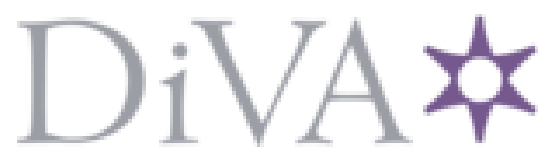

http://www.diva-portal.org

This is the published version of a paper published in Physical Review E. Statistical, Nonlinear, and Soft Matter Physics.

Citation for the original published paper (version of record):

Olsson, P., Teitel, S. (2013)

Athermal jamming versus thermalized glassiness in sheared frictionless particles.

Physical Review E. Statistical, Nonlinear, and Soft Matter Physics, 88(1): 010301

http://dx.doi.org/10.1103/PhysRevE.88.010301

Access to the published version may require subscription.

N.B. When citing this work, cite the original published paper.

Permanent link to this version:

http://urn.kb.se/resolve?urn=urn:nbn:se:umu:diva- 79250 


\title{
Athermal jamming versus thermalized glassiness in sheared frictionless particles
}

\author{
Peter Olsson $^{1}$ and S. Teitel ${ }^{2}$ \\ ${ }^{1}$ Department of Physics, Umeå University, 90187 Umeå, Sweden \\ ${ }^{2}$ Department of Physics and Astronomy, University of Rochester, Rochester, New York 14627, USA
}

(Received 12 November 2012; revised manuscript received 17 June 2013; published 2 July 2013)

\begin{abstract}
Numerical simulations of soft-core frictionless disks in two dimensions are carried out to study the behavior of a simple liquid as a function of temperature $T$, packing fraction $\phi$, and uniform applied shear strain rate $\dot{\gamma}$. Inferring the hard-core limit from our soft-core results, we find that it depends on the two parameters $\phi$ and $T / \dot{\gamma}$. Here $T / \dot{\gamma} \rightarrow 0$ defines the athermal limit in which a shear-driven jamming transition occurs at a well defined $\phi_{J}$ and $T / \dot{\gamma} \rightarrow \infty$ defines the thermalized limit where an equilibrium glass transition may take place at $\phi_{G}$. This conclusion argues that athermal jamming and equilibrium glassy behavior are not controlled by the same critical point. Preliminary results suggest $\phi_{G}<\phi_{J}$.
\end{abstract}

DOI: 10.1103/PhysRevE.88.010301

PACS number(s): 45.70.-n, 64.60.-i, 83.80.Fg

Different physical systems undergo a transition from a liquidlike state to a disordered solid state upon varying some external control parameter [1]. Athermal granular particles jam as the packing fraction $\phi$ increases above a critical $\phi_{J}$. Liquids may freeze into a glass as temperature $T$ decreases below the glassy $T_{g}$. Foams cease flowing as the applied shear stress $\sigma$ decreases below the yield stress $\sigma_{Y}$. Liu and co-workers [2,3] attempted to unite these phenomena into a single jamming phase diagram, with axes $\phi, T$, and $\sigma$.

In the equilibrium $\phi$-T plane, as $\phi$ decreases, the glassy $T_{g}(\phi)$ decreases and may vanish at a $\phi_{G}$. Liu and co-workers proposed that $\phi_{G}$ was the same as the athermal jamming $\phi_{J}$. The point $T=0, \phi=\phi_{J}, \sigma=0$ would thus locate a special critical point $J$ controlling not only athermal jamming, but also finite-temperature glassy behavior. However, restricting to the $\phi-T$ plane, it is generally accepted from mean-field calculations [4-7] and numerical simulations [8-11] that the location of $T=0$ jamming is protocol dependent. A continuous range of $\phi_{J}$ is found, depending upon the ensemble of initial states and on the rates of compression or cooling. Rapid quenching from random configurations gives a lower bound, often associated with random close packing. It remains unclear if a well-defined nontrivial upper bound exists or if crystallization must in principle occur for sufficiently slow rates [12]. A true equilibrium glass transition $T_{g}$, however, should be protocol independent. The connection between athermal jamming and an equilibrium glass transition is therefore subtle if one stays in the $\phi-T$ plane.

Here we address this problem by looking along the third axis of the phase diagram of Liu and co-workers, considering systems undergoing uniform steady state shear at a fixed applied shear strain rate $\dot{\gamma}$. Considering a model of overdamped, frictionless disks in two dimensions (2D), we have shown [9] that athermal steady state shearing defines a statistical ensemble of states, yielding a uniquely defined shear-driven jamming transition $\phi_{J}$ in the limit $\dot{\gamma} \rightarrow 0$, independent of the initial configuration. We now extend these investigations to finite $T$. Using dimensional arguments and inferring the hard-core limit from simulations of soft-core particles, we show that athermal jamming and thermal glassy behavior are given by opposite limits of a key control parameter of the hard-core system. We thus conclude that there is no reason to expect that these two phenomena are controlled by the same physical processes, nor any reason to expect $\phi_{G}=\phi_{J}$.

Our model is a bidisperse mixture of frictionless soft-core disks in $2 \mathrm{D}$, with equal numbers of large and small particles with diameter ratio $d_{l} / d_{s}=1.4$ [3]. The interaction between overlapping particles $i$ and $j$ is harmonic, $V\left(r_{i j}\right) \equiv \epsilon \tilde{V}\left(r_{i j}\right)=$ $\frac{1}{2} \epsilon \delta_{i j}^{2}$, where $\delta_{i j}=1-r_{i j} / d_{i j}$ is the relative particle overlap, $r_{i j}$ is the particles center to center distance, and $d_{i j}$ is the sum of their radii. We use overdamped dynamics [13] with a viscous dissipation with respect to an imposed average linear shear velocity flow in the $\hat{\mathbf{x}}$ direction,

$$
\lambda\left[\frac{d \mathbf{r}_{i}}{d t}-y_{i} \dot{\gamma} \hat{\mathbf{x}}\right]=-\sum_{j} \frac{d V\left(r_{i j}\right)}{d \mathbf{r}_{i}}+\zeta_{i},
$$

where $\lambda$ is the viscous damping coefficient. Temperature is modeled by a random Langevin thermal force $\zeta_{i}$ satisfying

$$
\left\langle\zeta_{i}\right\rangle=0, \quad\left\langle\zeta_{i}(t) \zeta_{j}\left(t^{\prime}\right)\right\rangle=2 \lambda T \delta_{i j} \delta\left(t-t^{\prime}\right) \mathbf{I},
$$

with I the identity tensor. We use Lees-Edwards boundary conditions [14] to apply a uniform shear strain rate $\dot{\gamma}$ to a box of length and height $L$ containing $N$ particles.

Defining the time constant $t_{0}=\lambda d_{s}^{2} / T$, we cast this equation of motion into dimensionless form by dividing each term by $T / d_{s}$ to get

$$
\frac{d \tilde{\mathbf{r}}_{i}}{d \tilde{t}}-\tilde{y}_{i}\left(\dot{\gamma} t_{0}\right) \hat{\mathbf{x}}=-\frac{\epsilon}{T} \sum_{j} \frac{d \tilde{V}}{d \tilde{\mathbf{r}}_{i}}+\tilde{\zeta}_{i},
$$

with dimensionless variables $\tilde{\mathbf{r}}_{i} \equiv \mathbf{r}_{i} / d_{s}, \tilde{t} \equiv t / t_{0}$, and noise $\tilde{\zeta}_{i}$ satisfying the correlation $\left\langle\tilde{\zeta}_{i}(\tilde{t}) \tilde{\zeta}_{j}\left(\tilde{t}^{\prime}\right)\right\rangle=2 \delta_{i j} \delta\left(\tilde{t}-\tilde{t}^{\prime}\right) \mathbf{I}$. In the hard-core limit $\epsilon / T \rightarrow \infty$, the first term on the righthand side provides an excluded-volume effect, preventing particle overlaps but introducing no energy or time scale. The sheared hard-core limit is thus entirely determined by two dimensionless parameters: the packing fraction $\phi$ and the Péclet number $\dot{\gamma} t_{0} \propto \dot{\gamma} / T$ (we will find it convenient to phrase our discussion in terms of the inverse Péclet number proportional to $T / \dot{\gamma}$ ). This immediately yields one of our main conclusions. The effects of temperature and shear on the hard-core system enter only via the combination $T / \dot{\gamma}$. Athermal jamming corresponds to $T \rightarrow 0$ first, followed by $\dot{\gamma} \rightarrow 0$, i.e., the limit $T / \dot{\gamma} \rightarrow 0$. Thermalized glassy behavior 
corresponds to $\dot{\gamma} \rightarrow 0$ first, followed by $T \rightarrow 0$, i.e., the limit $T / \dot{\gamma} \rightarrow \infty$. As these cases occur at the extreme opposite limits of the control parameter $T / \dot{\gamma}$, there is no reason to expect that jamming and the glass transition share a common physical mechanism or that they occur at the same value of the packing fraction $\phi$.

We now consider the shear viscosity $\eta \equiv \sigma / \dot{\gamma}$. In terms of the above dimensionless variables, the dimensionless viscosity $\tilde{\eta}$, with a well-defined hard-core limit, is $\tilde{\eta}=\eta / \lambda d_{s}^{2}$. By measuring the viscosity $\eta(\phi, \dot{\gamma}, T)$ of soft-core particles, we will infer the hard-core limit $\tilde{\eta}_{\mathrm{HC}}(\phi, T / \dot{\gamma})$. The athermal jamming transition is defined by the $\phi_{J}$ where $\tilde{\eta}_{\mathrm{HC}}\left(\phi_{J}, 0\right) \rightarrow$ $\infty$, while the hard-core glass transition is defined by the $\phi_{G}$ where $\tilde{\eta}_{\mathrm{HC}}\left(\phi_{G}, \infty\right) \rightarrow \infty$.

Our simulations are at fixed packing fraction $\phi$, using $N=$ 65536 particles so that finite-size effects are negligible for the parameters utilized in this work. The elastic part of the stress tensor $p_{\alpha \beta}$ is computed from the contact forces in the usual way [3]; the elastic part of the pressure is $p \equiv\left(p_{x x}+p_{y y}\right) / 2$ and the shear stress is $\sigma=p_{x y}$. We measure length in units such that $d_{s}=1$, energy in units such that $\epsilon=1$, and time in units such that $\lambda d_{s}^{2} / \epsilon=1$. In these units we have $\tilde{\eta}=\eta$. Similar shear-driven simulations at finite $T$ have been carried out for underdamped particles by others $[15,16]$.

We consider first a value of $\phi=0.72$, well below the jamming $\phi_{J} \approx 0.843$ [17]. In Fig. 1(a) we plot our results for $\eta$ vs $T$ for several different values of $\dot{\gamma}$. We also show the linear response $\eta_{\mathrm{GK}}$, computed using the Green-Kubo formula [14] applied to equilibrium ( $\dot{\gamma}=0)$ simulations of Eq. (1) [18]. We see that at high $T$ all the data collapse to a common curve; $\eta$ is independent of $\dot{\gamma}$, indicating a linear rheology where thermal fluctuations dominate over shear-induced fluctuations. As $T$ decreases, $\eta_{\mathrm{GK}}$ increases and saturates to a fixed value, which
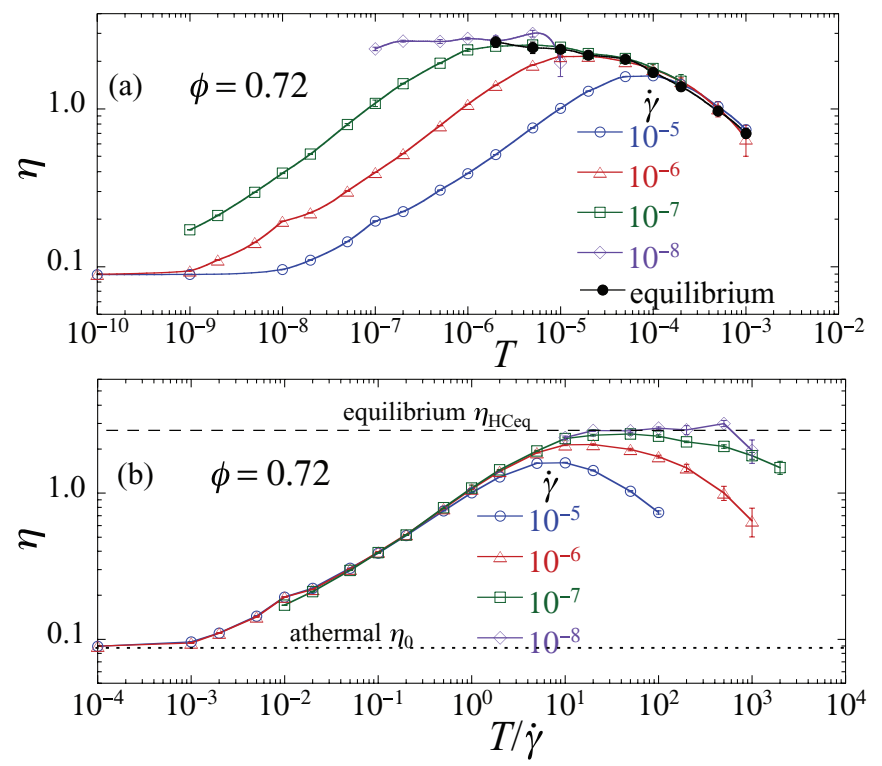

FIG. 1. (Color online) Shear viscosity $\eta$ vs (a) $T$ and (b) $T / \dot{\gamma}$, at fixed packing fraction $\phi=0.72$ below jamming, for several different shear strain rates $\dot{\gamma}$. In (a) solid dots are the linear response $\eta_{\mathrm{GK}}$ computed from the Green-Kubo formula in equilibrium. In (b) the horizontal dashed line is the hard-core equilibrium limit $\eta_{\mathrm{HCeq}}$; the horizontal dotted line is the athermal value $\eta_{0}$ at $T=0$. is just the hard-core viscosity in thermal equilibrium $\eta_{\mathrm{HCeq}}$. As $T$ decreases at finite $\dot{\gamma}$, however, $\eta$ increases to a peak value $\eta_{\text {peak }}(\phi, \dot{\gamma})$ at a temperature $T_{\text {peak }}(\phi, \dot{\gamma})$ and then decreases to a finite value as $T \rightarrow 0$. As $\dot{\gamma}$ decreases, $\eta_{\text {peak }}$ saturates to $\eta_{\text {HCeq }}$.

On the low- $T$ side of $\eta_{\text {peak }}$, the rheology is highly nonlinear ( $\eta$ varying with $\dot{\gamma}$ ) until converging to a common value as $T \rightarrow 0$. This $T=0$ value is just the athermal viscosity $\eta_{0}$. This low- $T$ side of $\eta_{\text {peak }}$ can be better understood by plotting $\eta$ vs $T / \dot{\gamma}$, as shown in Fig. 1(b). We now see that the data below $\eta_{\text {peak }}$ for different $\dot{\gamma}$ collapse to a common curve. This common curve, as $\dot{\gamma} \rightarrow 0$, represents the hard-core limit predicted by Eq. (3), which we see is a smooth sigmoidal-shaped curve increasing monotonically from $\eta_{0}$ in the athermal $T / \dot{\gamma} \rightarrow 0$ limit to $\eta_{\mathrm{HCeq}}$ in the thermalized $T / \dot{\gamma} \rightarrow \infty$ limit. Data at finite $\dot{\gamma}$ to the right of $\eta_{\text {peak }}(\dot{\gamma})$, which fall below this limiting $\dot{\gamma} \rightarrow 0$ curve, represent the parameter region where soft-core effects are important. From Fig. 1(a) we conclude that as $\dot{\gamma} \rightarrow 0$, the hard-core region of the system gets pushed down to $T \rightarrow 0$. The thermalized limit $\lim _{T \rightarrow 0}\left[\lim _{\dot{\gamma} \rightarrow 0} \eta\right]=$ $\eta_{\text {HCeq }}$ becomes singularly decoupled from the athermal limit $\lim _{\dot{\gamma} \rightarrow 0}\left[\lim _{T \rightarrow 0} \eta\right]=\eta_{0}$.

The nonmonotonic behavior of $\eta(T)$ at fixed $\dot{\gamma}$ can be understood as due to competing effects of thermal fluctuations on the hard-core vs the soft-core regions of the system behavior. As $T$ increases in the hard-core region, the forces associated with collisions increase and hence pressure $p$ increases. Since particles cannot pass through each other, it is difficult for shear stress to relax and so as $p$ increases, so does $\sigma$ and hence $\eta$. As $T$ increases further, however, one enters the soft-core region where there is enough thermal energy for particles to press into each other. Particles may now squeeze past each other, allowing for more rapid relaxation of shear stress, with a decrease in $\sigma$ and hence $\eta$.

It is interesting to consider how other quantities depend on the hard-core parameter $T / \dot{\gamma}$. In Fig. 2(a) we plot the inverse reduced pressure $n T / p$, with $n \equiv N / L^{2}$ the density of particles, vs $T / \dot{\gamma}$. In the thermalized limit $T / \dot{\gamma} \rightarrow \infty$ we see that the curves, as $\dot{\gamma} \rightarrow 0$, approach the hard-core equilibrium value, as we have computed independently from pair correlations [19] evaluated in equilibrium hard-core Monte Carlo (MC) simulations. In the athermal limit $T / \dot{\gamma} \rightarrow$ $0, n T / p \rightarrow 0$ as expected. In Fig. 2(b) we plot the stress anisotropy $\sigma / p$ vs $T / \dot{\gamma}$. In the thermalized $T / \dot{\gamma} \rightarrow \infty$ limit we find $\sigma / p \rightarrow 0$ as expected; as $\dot{\gamma} \rightarrow 0$, the thermalized system maintains a finite pressure but no shear stress. In the
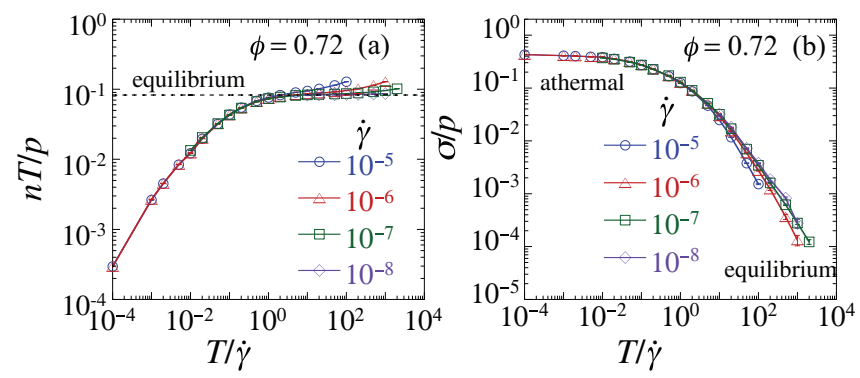

FIG. 2. (Color online) (a) Inverse reduced pressure $n T / p$ and (b) stress anisotropy $\sigma / p$ vs $T / \dot{\gamma}$, at packing fraction $\phi=0.72$ for several different shear strain rates $\dot{\gamma}$. The dashed horizontal line in (a) is the hard-core equilibrium value of $n T / p$. 

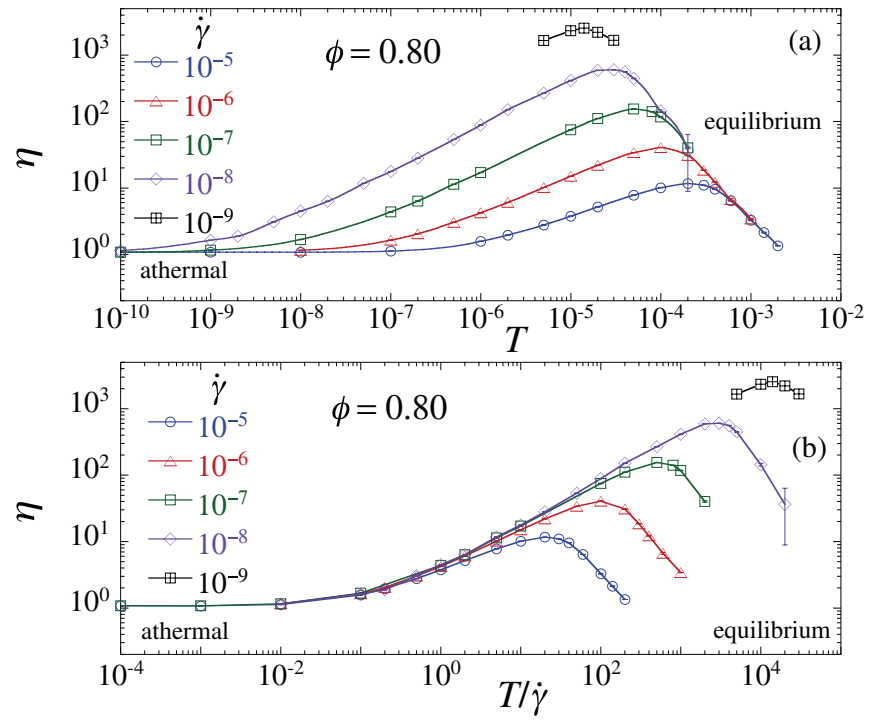

FIG. 3. (Color online) Shear viscosity $\eta$ vs (a) $T$ and (b) $T / \dot{\gamma}$, at fixed packing fraction $\phi=0.80$, closer to the jamming $\phi_{J}=0.843$, and several different shear strain rates $\dot{\gamma}$.

athermal $T / \dot{\gamma} \rightarrow 0$ limit, $\sigma / p$ approaches a finite value, as has been observed earlier [20]; in the athermal limit, both $\sigma$ and $p$ vanish as $\dot{\gamma} \rightarrow 0$, but do so in a way that their ratio becomes constant. This appearance of anisotropy as one moves from the thermalized to the athermal limit has recently been observed in experiments on colloidal particles [21].

Returning to viscosity $\eta$, we now consider behavior at higher packing fractions $\phi$. In Fig. 3 we show results for $\eta$ for different values of $\dot{\gamma}$ at the higher value of $\phi=0.80$. As in Fig. 1, we see that the data collapse on the high- $T$ side of $\eta_{\text {peak }}$ when plotted vs $T$, but collapse on the low- $T$ side of $\eta_{\text {peak }}$ when plotted vs $T / \dot{\gamma}$. Unlike Fig. 1 however, we see that $\eta_{\text {peak }}$ continues to increase as $\dot{\gamma}$ decreases, with no sign yet of saturating. Our data in this high- $T / \dot{\gamma}$ limit are not at sufficiently small $\dot{\gamma}$ to have reached the hard-core limit. Equilibrium simulations at this high $\phi$ cannot be sufficiently equilibrated to directly compute $\eta_{\mathrm{HCeq}}$.

That the hard-core viscosity $\eta_{\mathrm{HC}}(\phi, T / \dot{\gamma})$ appears to be monotonically increasing as $T / \dot{\gamma}$ increases and that,

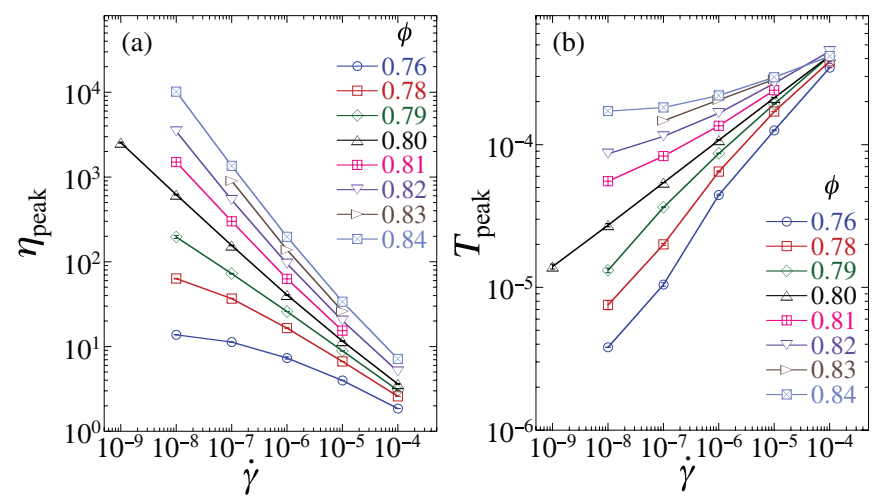

FIG. 4. (Color online) (a) Peak value of viscosity $\eta_{\text {peak }}$ and (b) location of the peak viscosity $T_{\text {peak }}$, vs strain rate $\dot{\gamma}$ for different packing fractions $\phi$. comparing Figs. 1 and $3, \eta_{\mathrm{HCeq}}$ appears to be increasing much more rapidly than $\eta_{0}$ as $\phi$ increases suggest a $\phi_{G}<\phi_{J}=$ 0.843 , in agreement with recent equilibrium simulations of soft-core particles in three dimensions [22,23]. In Fig. 4(a) we plot $\eta_{\text {peak }}$ vs $\dot{\gamma}$ for several different values of $\phi$, while in Fig. 4(b) we plot $T_{\text {peak }}$. Since, as $\dot{\gamma} \rightarrow 0, \eta_{\text {peak }} \rightarrow \eta_{\text {HCeq }}, \eta_{\text {peak }}$ should stay finite for $\phi<\phi_{G}$, while $\eta_{\text {peak }} \rightarrow \infty$ for $\phi \geqslant \phi_{G}$. Looking at the raw data in Fig. 4(a), such a change in behavior appears to happen at $\phi^{*} \approx 0.80$. In Fig. 4(b) $T_{\text {peak }}$ shows a similar marked change in behavior at the same $\phi^{*}$, with $T_{\text {peak }} \rightarrow 0$ as $\dot{\gamma} \rightarrow 0$ for $\phi \leqslant \phi^{*}$, while $T_{\text {peak }}$ is decreasing much more slowly, and perhaps saturating to a finite value, for $\phi>\phi^{*}$.

We can try to quantify this behavior with a critical scaling analysis. Assuming the usual algebraic scaling of a continuous critical point at $\phi_{G}$, we expect $\eta$ to satisfy a scaling relation

$$
\eta(\phi, T, \dot{\gamma})=b^{\beta / v} f\left(\delta \phi b^{1 / v}, \dot{\gamma} b^{z}, T b^{w}\right),
$$

where $\delta \phi \equiv \phi-\phi_{G}$ and $b$ is an arbitrary length rescaling factor [24]. The peak in $\eta$ as $T$ varies for fixed $\phi$ and $\dot{\gamma}$ will occur when the scaling function $f(x, y, z)$ has a maximum at some $z_{\text {peak }}(x, y)$. This leads to the scaling equation

$$
\eta_{\text {peak }}(\phi, \dot{\gamma})=b^{\beta / v} g\left(\delta \phi b^{1 / v}, \dot{\gamma} b^{z}\right)
$$

with $g(x, y) \equiv f\left(x, y, z_{\text {peak }}(x, y)\right)$. Choosing $b=\dot{\gamma}^{-1 / z}$ then gives

$$
\eta_{\text {peak }} \dot{\gamma}^{\beta / z v}=h\left(\delta \phi / \dot{\gamma}^{1 / z v}\right),
$$

with $h(x) \equiv g(x, 1)$. Expanding $h(x)$ as a polynomial in $x$, we fit our data in Fig. 4(a) to the scaling form of Eq. (6), keeping $\beta, z \nu, \phi_{G}$, and the polynomial coefficients as free fitting parameters. Considering only data with $\dot{\gamma} \leqslant 10^{-6}$, our fit yields the data collapse shown in Fig. 5(a), with fitted values $\phi_{G} \approx 0.796, \beta \approx 2.7$, and $z v \approx 5.1$. Choosing $b=|\delta \phi|^{-v}$ in Eq. (5) to get $\eta_{\text {peak }}=|\delta \phi|^{-\beta} g\left( \pm 1, \dot{\gamma} /|\delta \phi|^{z v}\right)$, we see that $\beta$ is just the exponent that describes the algebraic divergence of the thermalized hard-core viscosity as $\phi \rightarrow \phi_{G}$. The same estimate for $\phi_{G}$ was obtained in simulations by Henrich et al. [25], who considered the same bidisperse two-dimensional model system but used a thermostated dynamics for hard-core massive particles.
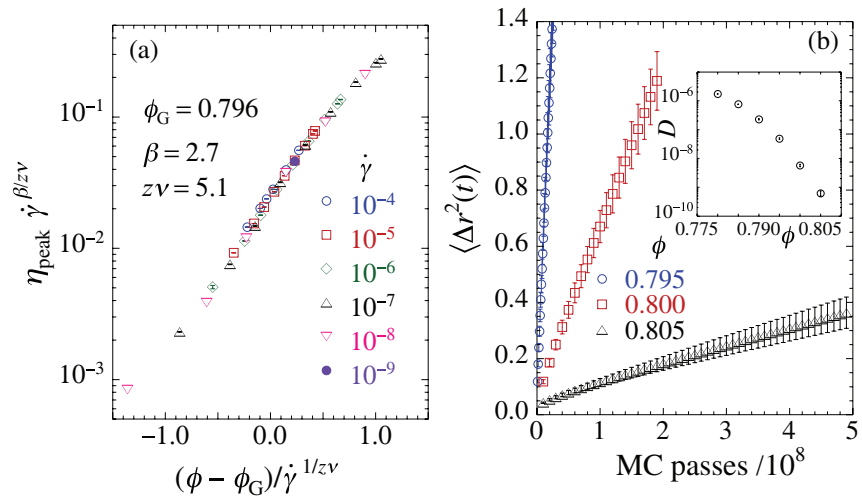

FIG. 5. (Color online) (a) Scaling collapse of $\eta_{\text {peak }}$ according to the scaling relation of Eq. (6). (b) Mean square displacement $\left\langle\Delta r^{2}\right\rangle$ vs MC passes for different $\phi$. The inset shows the diffusion constant $D$ vs $\phi$. 
Although our fit in Fig. 5(a) appears to look good, we find that the values of our fitted parameters are somewhat sensitive to the range of data that we use. As we restrict data to a smaller window of $\dot{\gamma}<\dot{\gamma}_{\max }$ the fitted value of $\phi_{G}$ appears to increase slightly, while the $\chi^{2} / \mathcal{D}$ decreases (where $\mathcal{D}$ denotes the degree of freedom). For our smallest window with $\dot{\gamma}_{\max }=$ $10^{-6}$ we find $\chi^{2} / \mathcal{D} \simeq 6$, suggesting a fit that is still less than ideal. Such behavior could be due to the effect of corrections to scaling, as we have previously shown to be important at the athermal jamming transition [17].

As an alternative approach to locating the thermalized $\phi_{G}$, we have carried out independent equilibrium $(\dot{\gamma}=0)$ MC simulations of hard-core disks. At each step of the simulation a particle is picked at random and displaced a distance $\delta \mathbf{r}$ with $\delta x, \delta y$ chosen from a uniform distribution on the interval $[-0.05,0.05]$. If no particle overlap results, the move is accepted; otherwise it is rejected. Here $N$ such steps constitutes one MC pass and represents one unit of time. With this MC dynamics we compute the single-particle mean square displacement $\left\langle\Delta r^{2}(t)\right\rangle \equiv(1 / N) \sum_{i=1}^{N}\langle| \mathbf{r}_{i}(t)-$ $\left.\left.\mathbf{r}_{i}(0)\right|^{2}\right\rangle$. In Fig. 5(b) we show our results for several different $\phi$. We see that $\left\langle\Delta r^{2}\right\rangle$ continues increasing with time for $\phi \lesssim 0.805$, suggesting a finite diffusion constant $D$, however $D$ is rapidly decreasing as $\phi$ increases [see inset in Fig. 5(b)]. These results thus suggest a $\phi_{G} \gtrsim 0.805$.

The discrepancy in the estimate of $\phi_{G}$ from our viscosity vs our diffusion data could be due to several factors: (i) the neglect of corrections to scaling in our analysis of $\eta_{\text {peak }}$, (ii) diffusion at the higher $\phi$ seems correlated with increased particle segregation and so may be reflecting an approach to a true phase-separated equilibrium [12] rather than a metastable glassy state, or (iii) it may be that the scaling shown in Fig. 5(a) reflects a transition that gets cut off by some other physical mechanism on longer time scales as $\dot{\gamma}$ decreases. We note that recent shearing simulations in three dimensions [26] similarly suggest a lower value for $\phi_{G}$ from viscosity measurements than was previously found from relaxation time measurements of both hard-core [27] and soft-core [22] particles in equilibrium [28]. The precise value of $\phi_{G}$ and a complete understanding of the thermal glass transition thus remain for future work.

To conclude, we have demonstrated that in the hard-core limit, a system of sheared overdamped particles is characterized not only by the packing fraction $\phi$, but by an additional control parameter $T / \dot{\gamma}$. The limit $T / \dot{\gamma} \rightarrow 0$ corresponds to the athermal limit, with a sharp jamming transition at $\phi_{J}$. The limit $T / \dot{\gamma} \rightarrow \infty$ corresponds to the thermalized limit, where equilibrium glassy behavior is observed. As the athermal and the thermalized regions are at opposite limiting values of $T / \dot{\gamma}$, there is no reason to expect athermal jamming and thermalized glassy behavior to be controlled by the same critical point. Our results find behavior consistent with a $\phi_{G}<\phi_{J}$. A recent work by Ikeda et al. [26] has reached similar conclusions.

We thank T. Haxton, A. J. Liu, and P. Schall for helpful discussions. We thank T. Marschall for the hard-core equilibrium simulations that yielded the equilibrium reduced pressure shown in Fig. 2(a). This work was supported by NSF Grant No. DMR-1205800 and Swedish Research Council Grant No. 2010-3725. Simulations were performed on resources provided by the Swedish National Infrastructure for Computing at PDC and HPC2N.
[1] H. M. Jaeger, S. R. Nagel, and R. P. Behringer, Rev. Mod. Phys. 68, 1259 (1996).

[2] A. J. Liu and S. R. Nagel, Nature (London) 396, 21 (1998).

[3] C. S. O'Hern, L. E. Silbert, A. J. Liu, and S. R. Nagel, Phys. Rev. E 68, 011306 (2003).

[4] F. Krzakala and J. Kurchan, Phys. Rev. E 76, 021122 (2007).

[5] R. Mari, F. Krzakala, and J. Kurchan, Phys. Rev. Lett. 103, 025701 (2009).

[6] G. Parisi and F. Zamponi, Rev. Mod. Phys. 82, 789 (2010).

[7] H. Jacquin, L. Berthier, and F. Zamponi, Phys. Rev. Lett. 106, 135702 (2011).

[8] P. Chaudhuri, L. Berthier, and S. Sastry, Phys. Rev. Lett. 104, 165701 (2010).

[9] D. Vågberg, P. Olsson, and S. Teitel, Phys. Rev. E 83, 031307 (2011).

[10] C. F. Schreck, C. S. O’Hern, and L. E. Silbert, Phys. Rev. E 84, 011305 (2011).

[11] M. Otsuki and H. Hayakawa, Phys. Rev. E 86, 031505 (2012).

[12] A. Donev, F. H. Stillinger, and S. Torquato, Phys. Rev. Lett. 96, 225502 (2006); J. Chem. Phys. 127, 124509 (2007).

[13] D. J. Durian, Phys. Rev. Lett. 75, 4780 (1995); Phys. Rev. E 55, 1739 (1997).

[14] D. J. Evans and G. P. Morriss, Statistical Mechanics of NonEquilibrium Liquids (Academic, London, 1990).
[15] T. K. Haxton, M. Schmiedeberg, and A. J. Liu, Phys. Rev. E 83, 031503 (2011).

[16] T. K. Haxton, Phys. Rev. E 85, 011503 (2012).

[17] P. Olsson and S. Teitel, Phys. Rev. E 83, 030302(R) (2011).

[18] For these equilibrium simulations we use only $N=64$ particles and even so we have found it exceedingly difficult to well equilibrate at the lowest temperatures.

[19] S. Luding and A. Santos, J. Chem. Phys. 121, 8458 (2004).

[20] See, P.-E. Peyneau and J.-N. Roux, Phys. Rev. E 78, 011307 (2008), which treats an athermal system with inertial effects; we have similarly found a finite value of $\sigma / p$ in an athermal overdamped model.

[21] V. Chikkadi, S. Mandal, B. Nienhuis, D. Raabe, F. Varnik, and P. Schall, Europhys. Lett. 100, 56001 (2012).

[22] L. Berthier and T. A. Witten, Europhys. Lett. 86, 10001 (2009); Phys. Rev. E 80, 021502 (2009).

[23] N. Xu, T. K. Haxton, A. J. Liu, and S. R. Nagel, Phys. Rev. Lett. 103, 245701 (2009).

[24] We note that mode coupling theories, while giving a power law divergence of $\eta$ below $\phi_{G}$, generally predict a discontinuous jump in yield stress at $\phi_{G}$ and so may be incompatible with a critical scaling model. See, M. Fuchs and M. E. Cates, Phys. Rev. Lett. 89, 248304 (2002); T. Voigtmann, Eur. Phys. J. E 34, 106 (2011).

[25] O. Henrich, F. Weysser, M. E. Cates, and M. Fuchs, Philos. Trans. R. Soc. London Ser. A 367, 5033 (2009). In this work, 
the authors analyze their data in terms of mode coupling theory and find qualitative but not quantitative agreement.

[26] A. Ikeda, L. Berthier, and P. Sollich, Phys. Rev. Lett. 109, 018301 (2012).

[27] G. Brambilla, D. El Masri, M. Pierno, L. Berthier, L. Cipelletti, G. Petekidis, and A. B. Schofield, Phys. Rev. Lett. 102, 085703 (2009).
[28] We note also that Refs. [22,27] claim an exponential divergence of the equilibrium relaxation time rather than the algebraic divergence that we and Ref. [26] find for the viscosity of shear-driven systems. We have tried to fit our present data for $\eta_{\text {peak }}$ to such an exponential form, however, we find such fits to generally be unstable and yield a larger $\chi^{2}$ error than our algebraic fit. 